\title{
Intermittency of two-dimensional decaying electron magnetohydrodynamic turbulence
}

\author{
G. Boffetta and A. Celani \\ Dipartimento di Fisica Generale, Università di Torino, Via Pietro Giuria 1, 1-10125 Torino, Italy \\ and Istituto Nazionale Fisica della Materia, Unità di Torino Università, Torino, Italy \\ A. Crisanti \\ Dipartimento di Fisica, Università “La Sapienza,", Piazzale Aldo Moro 2, I-00185 Roma, Italy \\ and Istituto Nazionale Fisica della Materia, Unità di Roma, Roma, Italy \\ R. Prandi \\ Dipartimento di Fisica Teorica, Università di Torino, Via Pietro Giuria 1, 10125 Torino, Italy \\ and Istituto Nazionale Fisica della Materia, Unità di Pisa, Pisa, Italy
}

(Received 6 October 1998)

\begin{abstract}
The intermittent nature of energy dissipation in two-dimensional electron-magnetohydrodynamic turbulence is investigated by means of high resolution direct numerical simulations. It is found that, when the main contribution to the energy is given by the magnetic field, dissipation is mostly concentrated on one-dimensional filaments. As a consequence, the multifractal spectrum has a simple form which can be approximately described in terms of a bifractal model. [S1063-651X(99)01803-6]
\end{abstract}

PACS number(s): 47.27.Eq

The statistical theory of three-dimensional fully developed hydrodynamic turbulence relies on one outstanding issue: the nonlinear transfer of energy from large to small scales $[1,2]$. The energy flux is constant over the intermediate scales of the inertial range, but does not need to be homogeneous in space. Moreover, experiments in fluid turbulence indicate that the self-similarity of the energy dissipation distribution is broken by the presence of small-scale structures in the flow. Recent direct numerical simulations revealed the presence of filaments and other dissipative structures candidate as physical sources of intermittency [3].

It is therefore interesting to look for two-dimensional turbulent systems sharing these same features. Actually many of them exhibit a reversed energy flux, from the small scales to the larger ones, as is the case of two-dimensional (2D) Navier-Stokes turbulence [4-7], Hasegawa-Mima turbulence [8], or its geophysical counterpart equivalent barotropic turbulence [9]. In this framework 2D electronmagnetohydrodynamic (EMHD) turbulence deserves special attention, beyond its modeling applications, since it has been shown to display, for the freely decaying case, a forward energy cascade á la Richardson-Kolmogorov [10].

EMHD equations are a fluid dynamical model for a cold electron plasma, moving in a uniform charge-neutralizing background of stationary ions. In recent years this model has received considerable interest for its relation to inertially confined plasma and to laser-plasma interactions, but the comparison with experimental results is limited by the fact that plasma which evolve according to EMHD equations are usually short-lived.

In the $2 \mathrm{D}$ case, the velocity and magnetic field are $z$ independent, and can be expressed in terms of the stream function $\varphi$ and the magnetic flux function $\psi$ according to $\boldsymbol{v}$ $=\left(-\partial_{y} \varphi, \partial_{x} \varphi,-\Delta \psi\right)$ and $\boldsymbol{B}=\left(-\partial_{y} \psi, \partial_{x} \psi, \varphi\right)$. The EMHD equation are then written for the scalar fields $\varphi$ and $\psi$ as

$$
\begin{aligned}
& \partial_{t}\left(\psi-d_{e}^{2} \Delta \psi\right)+\left[\varphi, \psi-d_{e}^{2} \Delta \psi\right]=-\mu_{\nu}(-\Delta)^{\nu} \psi \\
& \partial_{t}\left(\varphi-d_{e}^{2} \Delta \varphi\right)+\left[\varphi, \varphi-d_{e}^{2} \Delta \varphi\right]-\left[\psi, \psi-d_{e}^{2} \Delta \psi\right] \\
& \quad=-\mu_{\nu}(-\Delta)^{\nu} \varphi
\end{aligned}
$$

where the Jacobian operator is defined as usual $[a, b]$ $=\partial_{x} a \partial_{y} b-\partial_{y} a \partial_{x} b$. The equations have been adimensionalized with respect to the characteristic macroscopic length $L$, the typical magnetic field $B_{0}$, and the characteristic time $\tau$ $=m_{e} c /\left(e B_{0} d_{e}^{2}\right)$, and $d_{e}=\left[m_{e} c^{2} /\left(4 \pi e^{2} n L^{2}\right)\right]^{1 / 2}$ is the ratio of the inertial electron length scale to the integral scale $L$. The density of the number of electrons $n$ is assumed to be uniform according to the incompressibility of the velocity field $\boldsymbol{\nabla} \cdot \mathbf{v}=0$. The generalized dissipation operators correspond to resistivity for $\nu=1$ and to electron viscosity for $\nu$ $=2$.

In the ideal limit $\mu_{\nu}=0$, Eqs. (1) and (2) conserve the total energy (kinetic plus magnetic)

$$
E=\int d^{2} x\left(d_{e}^{2} \boldsymbol{v}^{2}+\boldsymbol{B}^{2}\right)
$$

For finite dissipation, EMHD exhibits a direct energy cascade from large to small scale, which suggests an analogy with 3D hydrodynamic turbulence. We thus expect, for a sufficiently small dissipation coefficient, a constant energy flux in an intermediate range of wave number (inertial range) where the dissipative effects can be neglected.

This suggests investigating in more detail the mechanism of energy transfer and energy dissipation to see whether there is any intermittency analogously to what observed in 3D hydrodynamic turbulence. Recently a relation analogous to the " $\frac{4}{5}$," Kolmogorov relation has been derived and tested for 2D-EMHD turbulence [11]. Let us recall that in 3D Navier-Stokes turbulence, the Kolmogorov law states that 
the average energy flux $\bar{\epsilon}=\langle\epsilon\rangle$ is related to velocity difference fluctuations $\partial v(\ell)$ at a scale $\ell$ by $\left\langle\delta v^{3}(\ell)\right\rangle$ $=-\frac{4}{5} \bar{\epsilon} \ell$. This relation is the starting point for the analysis of the scaling properties of the generic structure function $\left\langle\delta v^{p}(\ell)\right\rangle$. The self-similarity assumption implies $\left\langle\delta v^{p}(\ell)\right\rangle$ $\sim \ell^{p / 3}$, and deviations from this scaling are associated, via the refined similarity hypothesis, to intermittency in the energy dissipation [3].

In EMHD turbulence, the situation is complicated by the fact the energy flux is written as a combination of correlations involving fluctuation at scale $\ell$ of both the velocity and the magnetic field (see Ref. [11]). Thus it is not obvious in this case how to derive a prediction for the scaling of a single structure function, because there may be strong cancellation effects.

The situation becomes simpler if one considers the limits $\ell \ll d_{e} \sim 1$, where the energy flux is locally dominated by the kinetic contribution, or $d_{e} \ll \ell \ll 1$, dominated by the magnetic energy. Simple dimensional considerations suggest that in the kinetic case $\delta v(\ell) \simeq \ell^{1 / 3}$ and thus we expect a Kolmogorov-like spectrum

$$
E(k)=C \bar{\epsilon}^{2 / 3} k^{-5 / 3} .
$$

In the magnetic limit, the leading contribution to the flux involves structure functions containing two magnetic fluctuations and one velocity fluctuation. Recalling that, on a dimensional basis $\delta B(\ell) \sim \delta v(\ell) \ell$, in this case we expect

$$
E(k)=C^{\prime} \bar{\epsilon}^{2 / 3} k^{-7 / 3} \text {. }
$$

Predictions (4) and (5) are very well verified in direct numerical simulations $[10,11]$.

With this preliminary results in mind, it is natural to ask whether also in 2D-EMHD turbulence the energy transfer to the dissipative scales is intermittent as it is supposed to be in 3D hydrodynamic turbulence. To address this point we have performed a detailed numerical investigation on high resolution simulations of Eqs. (1) and (2) in the magnetic regime. We use a pseudospectral numerical code on a periodic $2 \pi \times 2 \pi$ box with $1024^{2}$ collocation points and a standard $\frac{2}{3}$ dealiasing rule. The physical parameters are $d_{e}=0.01, \nu$ $=3$, and $\mu_{3}=10^{-10}$. Following Ref. [10], the initial conditions are given by $\psi(k)=\exp \left[-k^{2} / 2 k_{0}^{2}+i \alpha(\boldsymbol{k})\right]$ and $\varphi(\boldsymbol{k})$ $=\exp \left[-k^{2} / 2 k_{0}^{2}+i \beta(\boldsymbol{k})\right]$, where $\alpha(\boldsymbol{k})$ and $\beta(\boldsymbol{k})$ are random phases and $k_{0}=5$. Since the initial energy is concentrated on large scales, we first observe a direct cascade toward small scales. The energy dissipation rate increases up to a maximum value at time $T_{m} \simeq 0.1$, at which we observe the spectrum (5) well developed on the inertial wave numbers $k$ $\leqslant d_{e}^{-1}$ (see Fig. 1).

As is customary, the intermittency of the energy cascade in the inertial range is studied by using the moments of the average energy dissipation [12]

$$
\left\langle\epsilon(\ell)^{p}\right\rangle=\left\langle\left[\frac{1}{V(\ell)} \int_{B(\mathscr{C})} d^{2} x \boldsymbol{\epsilon}(\mathbf{x})\right]^{p}\right\rangle,
$$

where $\epsilon(\boldsymbol{x})$ is the local energy dissipation and $B(\ell)$ is a box of size $\ell$ and volume $V(\ell)$ centered on $x$. For our simulations, $\langle\cdots\rangle$ stands for the spatial average. Of course, we have

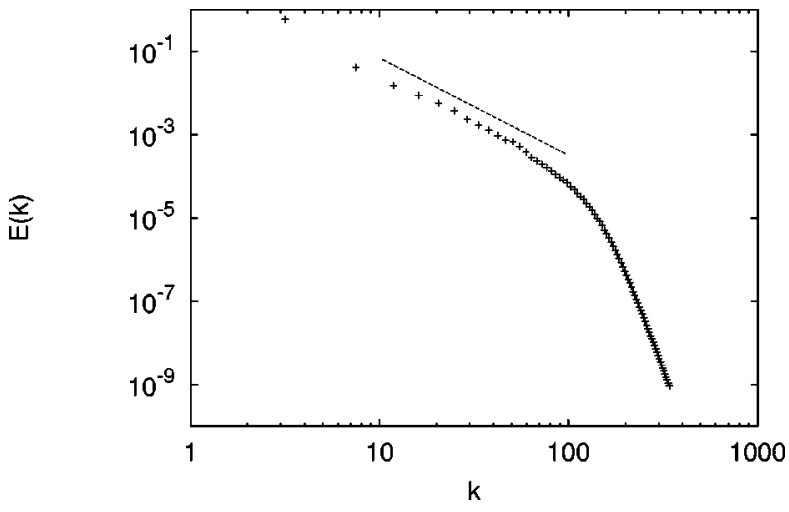

FIG. 1. Energy spectrum at $t=0.1$ for the decaying simulation at resolution $N=1024$. The line represent the theoretical spectrum $E(k) \sim k^{-7 / 3}$.

$\langle\epsilon(\ell)\rangle=\bar{\epsilon}$ for any $\ell$, while we expect a scale dependence for $p \neq 1$ in Eq. (6) if the dissipation is not uniformly distributed in space. The statistical properties of the dissipation fluctuations are summarized by the set of scaling exponents $\tau(p)$ defined by

$$
\left\langle\epsilon(\ell)^{p}\right\rangle \sim \ell^{\tau(p)} .
$$

In the multifractal description of intermittency, the scaling exponents $\tau(p)$ are given in terms of a Legendre transformation

$$
\tau(p)=\min _{\alpha}[q \alpha+2-F(\alpha)],
$$

where $\alpha$ is the scaling exponent of local energy dissipation which is realized on a set of dimension $F(\alpha) \leqslant 2$.

In Fig. 2, we plot the spectrum $\tau(p)$ obtained from the fit of $\left\langle\epsilon(\ell)^{p}\right\rangle$ in our simulations. The most remarkable feature is the linear dependence on $p$ for almost all $p$ :

$$
\begin{array}{cc}
\tau(p) \simeq 0.2 p \quad \text { for } p<0 \\
\tau(p) \simeq-1.2 p & \text { for } p>2 .
\end{array}
$$

Our findings have a simple physical interpretation if we look at the snapshot of the energy dissipation field $\boldsymbol{\epsilon}(\boldsymbol{x})$, like

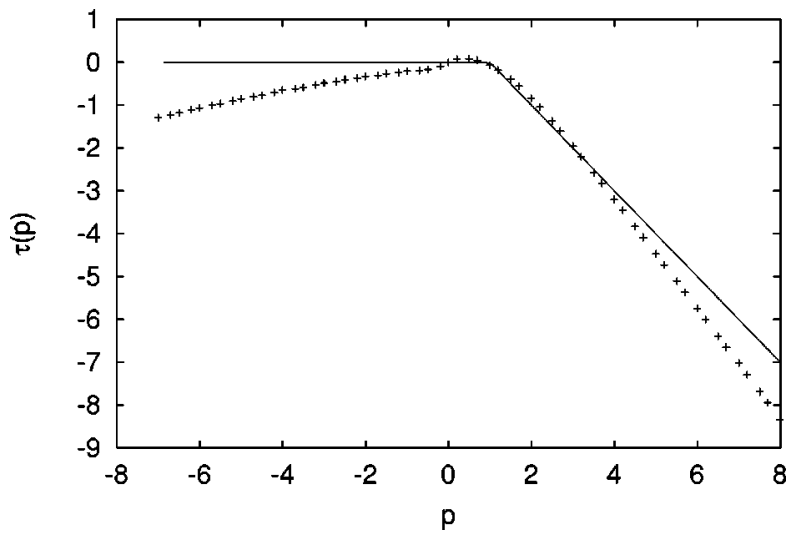

FIG. 2. Energy dissipation scaling exponents $\tau(p)$ obtained by a $\log -\log$ fit of the $\epsilon(l)$. The continuous line represents the prediction of the bifractal model as discussed in the text. 


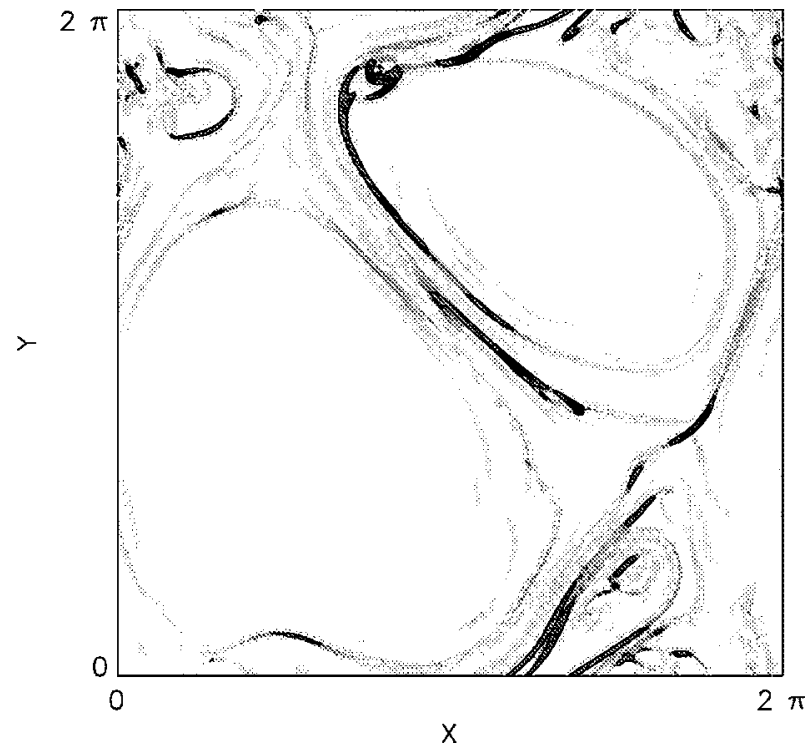

FIG. 3. Instantaneous energy dissipation field $\epsilon(x)$ at $t=0.1$. Gray indicates dissipation active regions.

that shown in Fig. 3. The most dissipative structures are the long filaments which cross all the domain, while there are large regions-corresponding to coherent structures in the magnetic field - in which the dissipation is almost zero. This suggests that a simple (bi)fractal model should be able to capture the main qualitative features of intermittency.

Let us divide the physical domain, which concerns the energy dissipation, into two sets: a background, twodimensional, region of almost no dissipation on which $\epsilon(\boldsymbol{x})$ $=\epsilon_{0}$; and an active one-dimensional region on which $\epsilon(\boldsymbol{x})$ $=\epsilon_{1} \gg \epsilon_{0}$. The average energy dissipation [Eq. (6)] is simply evaluated as $\epsilon(\ell) \sim \epsilon_{0}$ if the box $B(\ell)$ does not contain dissipation filaments, while we have $\epsilon(\ell) \sim \epsilon_{1} / \ell$ if $B(\ell)$ includes a dissipative filament. As $\ell \rightarrow 0$, the probabilities of finding the two sets scale respectively as $p(\ell) \sim \ell^{0}$ and $p(\ell) \sim \ell$; thus we can write

$$
\left\langle\epsilon(\ell)^{p}\right\rangle \sim\left(\frac{\epsilon_{1}}{\ell}\right)^{p} \ell+\epsilon_{0}^{p} \sim \epsilon_{1}^{p} \ell^{1-p}+\epsilon_{0}^{p} .
$$

For $\ell \rightarrow 0$ the leading contribution in Eq. (11) is selected by the value of $p$, so that the scaling exponents predicted by the bifractal model are

$$
\begin{gathered}
\tau(p)=0 \quad \text { for } p \leqslant 1, \\
\tau(p)=1-p \quad \text { for } p \geqslant 1 .
\end{gathered}
$$

Figure 2 shows that predictions (12) and (13) are only qualitatively correct. The deviation from the numerical data cannot be explained on the basis of statistical errors, i.e., the numerical uncertainty of the scaling exponents is very small. It is relatively simple to correct the prediction for $\tau(p)$ by playing with the parameters of the bifractal model, but the physical interpretation becomes less clear. For positive $p$ the fact that $\tau(p) \sim-1.2 p$ instead of $\tau(p) \sim-p$ can be explained by assuming that the set on which the dissipation is most active has a fractal dimension slightly less than 1 .

It is interesting to compare the present situation with the other renowned turbulent model displaying bifractality, i.e., the Burgers equation. In that case bifractal scaling is an exact consequence of the infinite number of conserved quantities [3], while here it is only approximate and does not have such a simple explanation. Despite these differences, in both cases bifractality reveals a strong intermittent statistics.

In conclusion, we have computed the multifractal scaling exponent of the energy dissipation for 2D-EMHD turbulence in the limit of negligible electron inertia. We have found fairly clear scaling for the local average of the energy dissipation, $\left\langle\epsilon(\ell)^{p}\right\rangle \sim \ell^{\tau(p)}$, with nontrivial scaling exponents $\tau(p)$ revealing a strong intermittency. We have shown that the main intermittency features can be captured by a bifractal model suggested by physical arguments.

This work was partially supported by the CNR Special Project 'Fully Developed Turbulence in Plasmas,', and by MURST (Program No. 9702265437).
[1] A. N. Kolmogorov, Dokl. Akad. Nauk SSSR 32, 16 (1941) [reprinted in Proc. R. Soc London, Ser. A 434, 15 (1991)].

[2] G. K. Batchelor, The Theory of Homogeneous Turbulence (Cambridge University Press, Cambridge, 1953).

[3] U. Frisch, Turbulence. The Legacy of A. N. Kolmogorov (Cambridge University Press, Cambridge, 1995).

[4] J. C. McWilliams, J. Fluid Mech. 146, 21 (1984).

[5] M. Brachet, M. Meneguzzi, H. Politano, and P. Sulem, J. Fluid Mech. 194, 33 (1988).

[6] B. Legras, P. Santangelo, and R. Benzi, Europhys. Lett. 5, 37 (1988).
[7] L. M. Smith and V. Yakhot, Phys. Rev. Lett. 71, 352 (1993).

[8] N. Kukharkin, S. A. Orszag, and V. Yakhot, Phys. Rev. Lett. 75, 2486 (1995).

[9] V. D. Larichev and J. C. McWilliams, Phys. Fluids A 3, 938 (1991).

[10] D. Biskamp, E. Schwarz, and J. F. Drake, Phys. Rev. Lett. 76, 1264 (1996).

[11] A. Celani, R. Prandi, and G. Boffetta, Europhys. Lett. 41, 13 (1998).

[12] A. M. Obukhov, J. Fluid Mech. 13, 77 (1962). 